\title{
Roller compaction of rock-fill with automatic frequency control
}

Carl Wersäll PhD

Researcher, Department of Civil and Architectural Engineering, KTH Royal Institute of Technology, Stockholm, Sweden (corresponding author: cwersall@kth.se) (Orcid:0000-0002-7361-0729)

Ingmar Nordfelt MSc

Research Manager, Dynapac Compaction Equipment AB, Karlskrona Sweden
Stefan Larsson PhD

Professor, Department of Civil and Architectural Engineering, KTH Royal Institute of Technology, Stockholm, Sweden (Orcid:0000-0001-9615-4861)

\begin{abstract}
A new intelligent compaction method for vibratory soil compaction rollers that automatically and continuously adjusts the vibration frequency to obtain resonance in the coupled roller-soil system was evaluated in full-scale field tests on a $1 \mathrm{~m}$ high rock-fill embankment. This method has been called automatic frequency control (AFC). Compacting at the resonant frequency results in maximised deformations in the soil by optimising the dynamic behaviour of the system and improving the interaction between the drum and the soil. The embankment was also compacted conventionally at a fixed standard frequency in order to quantify the difference between the two methods. Applying AFC resulted in a higher degree of compaction of the embankment layer. The effect was most significant at the top of the embankment, where considerable compression was obtained - in contrast to the very loose material resulting from conventional compaction - thus eliminating the need for subsequent static passes. Spot tests showed a considerably stiffer embankment surface after a high number of passes using AFC. Other observed benefits of AFC include a more homogeneously compacted embankment, which benefits long-term behaviour, up to $15 \%$ reduced fuel consumption, reduced environmental impact and less machine wear.
\end{abstract}

\author{
Notation \\ $A_{\Omega} \quad$ spectral amplitude at the fundamental excitation \\ frequency \\ $A_{0.5 \Omega} \quad$ spectral amplitude at half the fundamental excitation \\ frequency \\ $E_{\mathrm{v} 2} \quad$ reloading modulus in plate load test \\ $m_{\mathrm{e}} e \quad$ eccentric moment \\ $P \quad$ centrifugal force \\ $\omega \quad$ circular frequency
}

\section{Introduction}

Vibratory rollers for the compaction of granular soils utilise a rotating eccentric mass to generate vibrations that cause the drum to vibrate and dynamically load the soil, producing cyclic shear stress and, in turn, cyclic shear strain within the soil mass. This process rearranges the soil particles into a denser state, which is the fundamental process of soil compaction. Since the early days of vibratory compaction, discussions have occurred regarding which roller parameters - whether the displacement amplitude, static linear load, acceleration, centrifugal force or some other property - best govern the compaction potential. Arguments have often been put forward based on empirical data investigating the influence of a single parameter, but no consensus regarding the optimum compaction parameters has been reached. The authors believe that this is mainly due to failure to regard the interaction between the roller and the soil as a dynamic system in which all parameters interact to determine the behaviour of the system. Thus, it is not possible to observe the influence of a single parameter independently. In a dynamic system - in this case the coupled roller-soil system - the behaviour is determined by stiffness, mass, load, frequency and non-linearity. The aim should be to obtain a combination of parameters that maximises deformations - and thus shear strain - in the soil material, since shear strain is the governing parameter for the rate of compaction (Arnold and Herle, 2009; Youd, 1972). The centrifugal force of the drum has long been regarded as a significant parameter. However, the load that governs deformations in the soil is the interaction force between the roller and the soil, which must be distinguished from the centrifugal force caused by the rotating mass in the drum.

The most fundamental parameter in any dynamic system is the frequency, with the relation between the excitation and resonant frequencies determining the state - and thus the behaviour - of the system. Below resonance, deformations are low and the load and displacement move in phase. Far above resonance, deformations are low but the load and displacement move out of phase. At resonance, however, the interaction between load and displacement is optimised to produce the largest deformations. In many applications, the aim is to minimise deformations, so resonance should be avoided. Such applications include, for example, environmental vibrations from traffic or construction activities. For compaction, however, the opposite is desirable, which motivates compaction at the resonant frequency. Rollers normally operate far above the resonance of a coupled roller-soil system and thus adjusting the frequency to resonance implies a significant frequency reduction. 
The concept of vibratory soil compaction at the resonant frequency was first proposed in the 1950s and was shown to be efficient in both laboratory tests (Bernhard, 1952) and field tests (Converse, 1953). However, due to the technical limitations of detecting resonance and adjusting the frequency, the concept of resonant compaction was not implemented in the infancy of vibratory soil compaction. Forssblad (1965) stated that it might be possible to utilise the effect of resonance at compaction, but that the advantages could not outweigh the technical difficulties and costs. Although this statement was likely correct at the time, it is definitely not true today. Modern technology has facilitated developments such as the application of roller-integrated acoustic wave detectors to evaluate soil stiffness (Zhang et al., 2019a) and unmanned roller navigation (Zhang et al., 2019b). Other developments include the application of artificial intelligence to roller compaction (Imran et al., 2018) and further extensions of continuous compaction control (CCC) techniques based on new technology (e.g. Liu et al., 2014; Liu et al., 2016; Meehan et al., 2017). Electronic measurement and control systems have made advanced control methods readily available. In the 1990s, the concept of resonant compaction was successfully introduced in deep compaction using vibratory probes (Massarsch, 1991; Massarsch and Fellenius, 2002). For roller compaction, however, the vibration frequency of the drum has remained significantly higher than the resonant frequency, up to the present day. Hypothesising that compaction could be made more efficient at the resonant frequency, a series of small-scale tests was conducted in a laboratory environment using electrodynamic vibrators (Wersäll and Larsson, 2013) and rotating mass oscillators (Wersäll et al., 2015). The results showed that resonance could be utilised to enhance the compaction of sand beds of varying thicknesses and moisture content. A subsequent series of full-scale tests using a modified vibratory roller under controlled indoor conditions showed that the coupled roller-soil resonant frequency is the most energy-efficient compaction frequency (Wersäll et al., 2017, 2018a), producing a higher compacted stiffness while reducing fuel consumption and machine wear. Based on these results, a new automated compaction method was developed. The technique - termed automatic frequency control (AFC) - automatically and continuously adjusts the vibration frequency of the drum to obtain resonance in the system, thus providing the largest possible deflection (i.e. displacement amplitude) of the drum and deformations within the soil. Automatic feedback and frequency adjustment to resonance for soil compaction rollers have previously been discussed in the literature (Anderegg, 2000; Anderegg and Kaufmann, 2004; White et al., 2004) but not verified in practice. Automatic frequency adjustment based on feedback from continuous measurements is commercially available in the Ammann Compaction Expert system (Anderegg et al., 2006) but is combined with adjustment of amplitude and does not lower the frequency sufficiently to obtain the maximum effect of resonance. It is notable that, since the resonant frequency depends heavily on both roller and soil properties and soil variations result in a high spatial variation of the resonant frequency (Mooney and Rinehart, 2007), the use of manual frequency adjustment to obtain resonance would bring great difficulties. Furthermore, an automatic system can ensure that compaction is never conducted below the resonant frequency, which has been shown to have a strong negative impact on the degree of compaction (Dobry and Whitman, 1973; D’Appolonia et al., 1969; Wersäll et al., 2017).

The first full-scale field tests investigating compaction efficiency using the AFC system for vibratory rollers are described in this paper. The tests were conducted on a trial embankment consisting of sub-ballast rock-fill material for railways (similar to sub-base material in road embankments), with a vibratory soil compaction roller equipped with the first implementation of the AFC system. In order to quantify the outcome of the AFC application, the embankment was divided into two parts - one was compacted using AFC and the other was compacted conventionally using a fixed frequency. Compaction and roller behaviour were evaluated by a combination of static and dynamic measurement sensors in the embankment and mounted on the roller, in combination with conventional in situ spot tests. The results of this study have previously been summarised in an extended abstract (Wersäll et al., 2018b).

\section{Experimental setup}

Field experiments were conducted adjacent to the new freight railway line Kardonbanan in Norrköping, Sweden, on a trial embankment of height $1 \mathrm{~m}$ after compaction. The material was crushed rock with a fraction of $0-150 \mathrm{~mm}$ according to Swedish regulations for sub-ballast material (Svensk Byggtjänst, 2013). Sieve analysis of the rock-fill is shown in Figure 1. The embankment, shown in Figure 2, was compacted in a single layer, although it was placed in two layers to

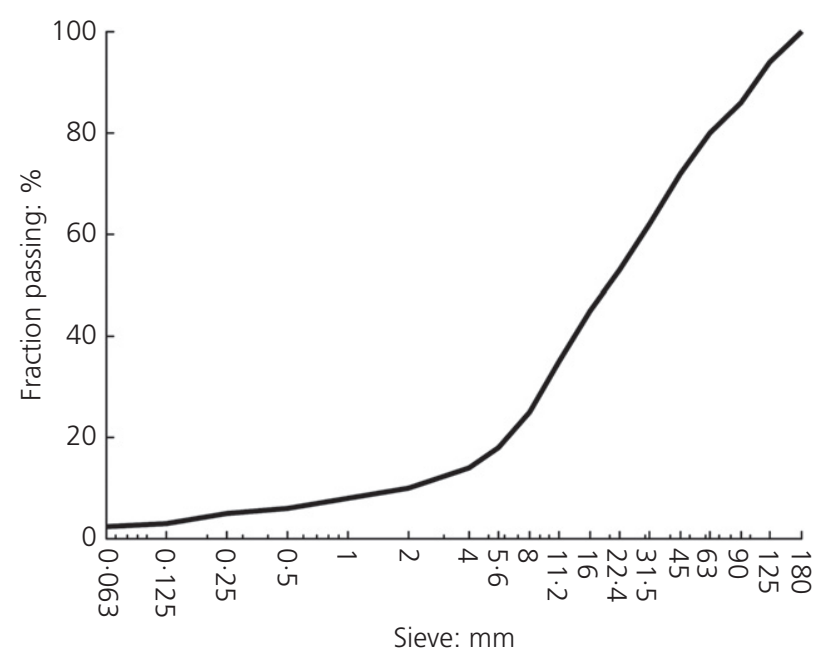

Figure 1. Sieve analysis of the rock-fill material 
Roller compaction of rock-fill with

automatic frequency control

Wersäll, Nordfelt and Larsson

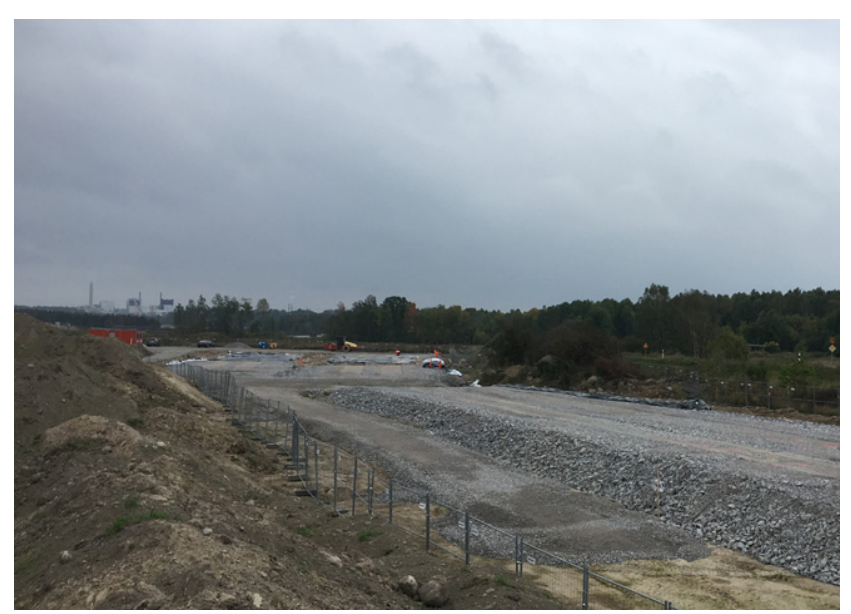

Figure 2. Trial embankment

facilitate the installation of measurement sensors. Placement was conducted using a bulldozer. Construction traffic caused some initial compaction at the surface of the bottom half of the layer as well as at the top of the layer. The subsoil below the embankment consisted of gravelly silty till. During the preparatory works, large amounts of rainfall saturated the subsoil material closest to the ground surface and, due to its high silt content (around 35\%), this resulted in a slightly softer material that was more prone to settlement. The subsoil was compacted using a roller until no progress in the compaction meter value was observed; this step was then followed by four static passes. Static plate load tests (PLTs) on the subsoil after compaction showed an average reloading modulus $\left(E_{\mathrm{v} 2}\right)$ of $65 \mathrm{MPa}$.

A prototype roller was developed that could operate either conventionally with a fixed frequency or using AFC with a variable frequency. The roller, shown in Figure 3, was based on a Dynapac CA6500D smooth-drum soil compaction roller

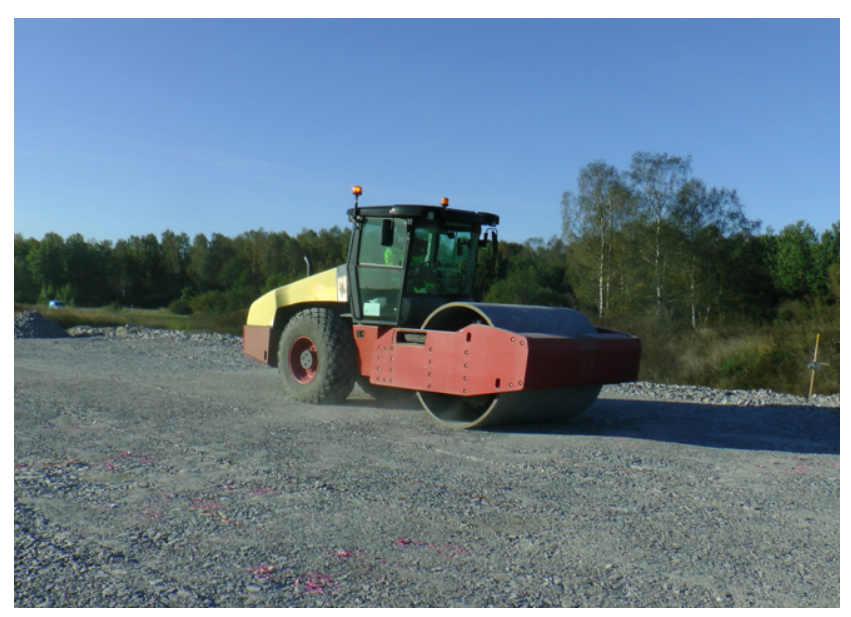

Figure 3. Prototype roller with a static linear load of $65 \mathrm{kN} / \mathrm{m}$ and a total weight of $20900 \mathrm{~kg}$. All tests were conducted at high amplitude, where the nominal amplitude was $2 \cdot 1 \mathrm{~mm}$. The tests operating with conventional compaction were conducted at a fixed standard frequency of $28 \mathrm{~Hz}$, while the AFC setting enabled a variable frequency in the range $17-28 \mathrm{~Hz}$. The resonant frequency of the roller-soil system was detected by an accelerometer integrated in the drum, and the compaction frequency was automatically adjusted so that the system was always close to a state of resonance. All passes were conducted with a roller speed of $1 \mathrm{~m} / \mathrm{s}$.

The surface of the embankment was $10 \mathrm{~m}$ wide and $28 \mathrm{~m}$ long. To facilitate a comparison of the two compaction methods, the surface was not compacted over its full width; instead, it was compacted in two parallel compaction lanes, with one lane compacted with AFC and the other compacted conventionally at a fixed frequency. The lanes were spaced $2 \mathrm{~m}$ apart from edge to edge and were $2 \cdot 13 \mathrm{~m}$ wide, the same width as the drum width. Since the spacing between the lanes was twice the height of the embankment, it was assumed to be sufficient to prevent lateral soil displacement from affecting the adjacent lane. Furthermore, the stress increase below the adjacent lane was less than $0 \cdot 1 \%$ of the applied load (calculated by Boussinesq stress distribution theory), implying no direct compaction on the adjacent lane. First, the lane dedicated for AFC was compacted in two passes, followed by two passes in the other lane with fixed frequency. This alternating compaction procedure was repeated with increments of 2-8 passes, changing the lane and compaction method after $2,4,8,12,16,20,24$ and 32 passes. The instrumented parts of the compaction lanes were $20 \mathrm{~m}$ long and a total of 32 passes was completed for each of the methods.

The settlement of the surface was measured at 63 points per lane using a total station after $0,2,4,8,12,16,20,24$ and 32 passes, resulting in a total of 1134 settlement measurements. PLTs were conducted in accordance with the German standard DIN 18134 (DIN, 2012) at eight points per lane after 8 and 32 passes, resulting in a total of 32 tests. Falling weight deflectometer (FWD) measurements were conducted in four locations along each lane after 32 passes applying loads of $30 \mathrm{kN}$ and $50 \mathrm{kN}$, resulting in a total of 16 tests. The plate diameter was $300 \mathrm{~mm}$ for both the PLTs and the FWD measurements. Spot testing by the PLTs and FWD measurements were conducted at different locations along the centre of the compaction lanes. Since soil properties along an embankment are somewhat spatially autocorrelated (Cai et al., 2019), spot tests were evenly spread out to minimise correlation within the sample. Deformations within the embankment were measured using inductance coil sensors (Janoo et al., $1999)$ called epsilon measuring unit (Emu) sensors. The individual coils consisted of copper wire wrapped numerous times around a circular disc. Several coils were buried in vertical alignment within the embankment material with a vertical 
spacing of $150-250 \mathrm{~mm}$. Inducing an electric current in one coil produced a magnetic field, which was detected by adjacent coils and could be correlated to the distance between them. With this procedure, the deformation of each $150-250 \mathrm{~mm}$ interval could be analysed throughout the compaction process, giving the depth-dependent compression within the embankment. Each lane had two columns of six coils, with the top sensor placed $30-50 \mathrm{~mm}$ below the embankment surface and the bottom sensor placed on the subsoil level. Deformation readings were conducted after $0,2,4,8,12,16,20,24$ and 32 passes. Installation of the Emu sensors in the uncompacted embankment is shown in Figure 4. A small amount of finer material $(0-16 \mathrm{~mm})$ was placed around each coil in order to obtain sufficient interaction between the sensor and the surrounding embankment material. The metal bar running through the centre of the coils facilitated vertical alignment and was removed after installation.

The roller characteristics were determined by integrated sensors, with the most important sensor being the accelerometer mounted on the drum. The accelerometer measured the acceleration during compaction, which was double-integrated to obtain the displacement amplitude. The roller also had an on-board GPS and measured the angular position of the eccentric mass in the drum. Combining these measurements is the basis for stiffness evaluation by CCC. The maximum contact force between the drum and the soil could be estimated by analysing the force-displacement relation, which was obtained from the accelerometer data, and the eccentric position. The accelerometer data also provided the resonance meter value (RMV), which is a measure of the amount of double-jumping experienced by the drum. A double-jump means that the time period in which the drum loses contact with the soil is longer than one cycle of vibration, which is not beneficial for compaction and roller behaviour (Adam, 1996). The RMV is expressed as a percentage and is

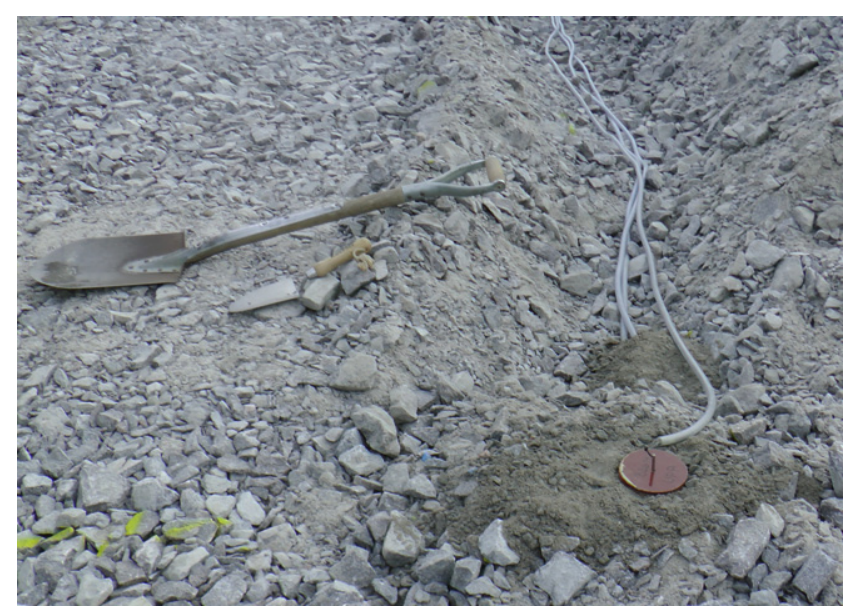

Figure 4. Installation of Emu sensors calculated using

1. $\mathrm{RMV}=100 \cdot \frac{A_{0.5 \Omega}}{A_{\Omega}}$

where $A_{\Omega}$ is the spectral amplitude at the fundamental excitation frequency and $A_{0.5 \Omega}$ is the spectral amplitude at half the fundamental excitation frequency. Double-jumping, which corresponds to a high RMV, should be avoided. Some rollers have an active protection system that either shuts down vibrations or indicates to the operator to do so manually and to switch to a low amplitude. High frequency, amplitude and soil stiffness all increase the risk of double-jumping.

\section{Results and discussion}

The results were divided into the parameters obtained by integrated roller measurements (i.e. accelerometer data and angular eccentric position), the results obtained by direct settlement and deformation measurements (i.e. total station and Emu sensors) and the stiffness spot tests (i.e. PLTs and FWD measurements). The effect of AFC on fuel consumption and machine wear is discussed at the end of this section. The application of CCC while using AFC was not evaluated in this paper, but has been studied by Wersäll et al. (2019), who reported that $\mathrm{CCC}$ can be used with $\mathrm{AFC}$ without modification.

\subsection{Integrated roller measurements}

The AFC system automatically ensures maximum deformation by continuously adjusting the compaction frequency. Since the resonant frequency depends on varying soil stiffness, the frequency and thus the displacement amplitude will also vary throughout the compaction process. The displacement amplitude is henceforth called the 'amplitude', which is common practice in the field of vibratory compaction. Figure 5 shows

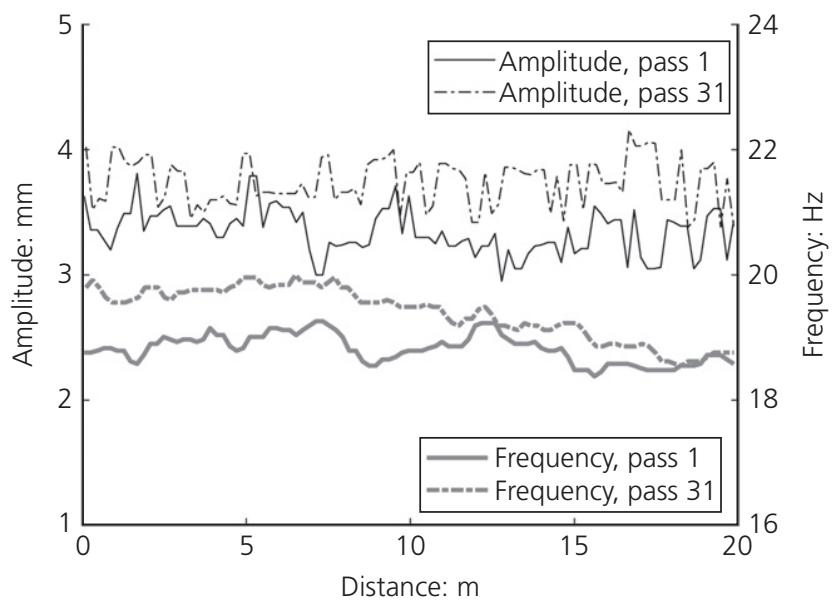

Figure 5. Frequency and amplitude variation during single passes using AFC 
the variation of the amplitude and frequency in passes 1 and 31 over the $20 \mathrm{~m}$ compaction lane using AFC. As the soil became more compacted - and thus stiffer - with a higher number of passes, both the resonant frequency and the amplitude increased slightly.

Figure 6(a) shows the minimum, average and maximum frequencies in every pass for AFC compared to conventional compaction at a constant frequency of $28 \mathrm{~Hz}$. Since AFC automatically adjusts the frequency to approach resonance and since conventional compaction is conducted far above resonance, AFC implies a significant reduction in frequency. The adjusted frequency is somewhat affected by the driving direction ( forwards = odd passes; backwards = even passes) since the interpreted resonant frequency depends to some extent on the rotation direction of the eccentric mass in relation to the rotation of

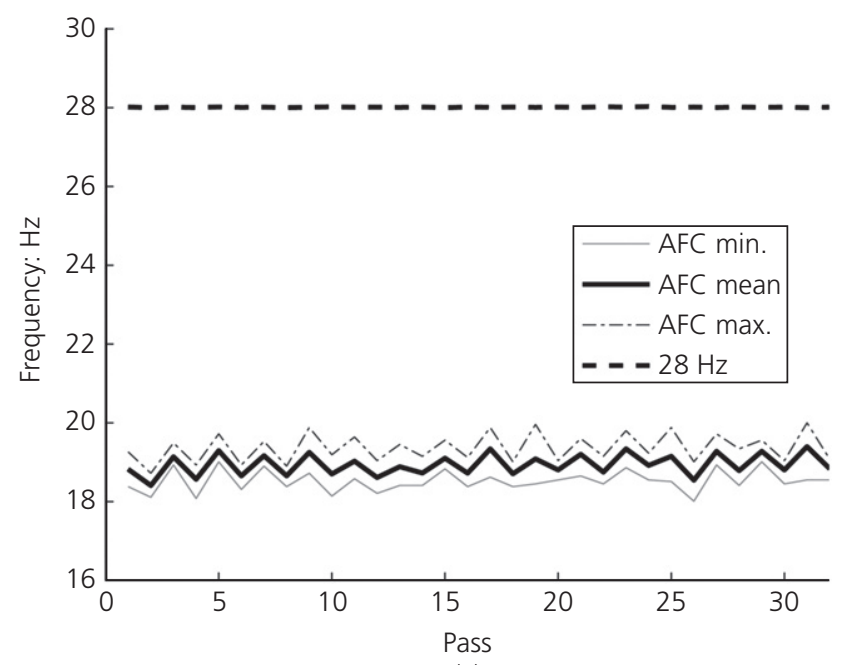

(a)

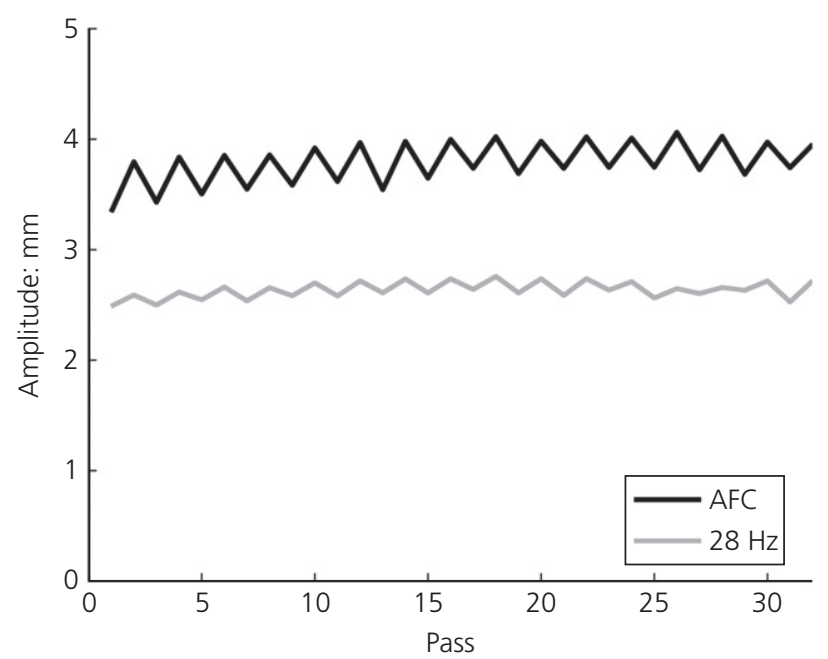

(b)

Figure 6. (a) Minimum, average and maximum frequencies for all passes; the frequency was fixed at $28 \mathrm{~Hz}$ during conventional compaction; (b) average amplitude for all passes the drum. The resulting average amplitudes of the two methods are shown in Figure 6(b). The large increase in amplitude for AFC, which is the result of resonance, is remarkable considering the significantly reduced centrifugal force. Equation 2 describes how the centrifugal force $(P)$ correlates with the circular frequency $(\omega)$, where $m_{\mathrm{e}} e$ is the eccentric moment.

2. $P=m_{\mathrm{e}} e \cdot \omega^{2}$

The eccentric moment of a CA6500D roller is $10 \cdot 31 \mathrm{~kg} . \mathrm{m}$, which implies centrifugal forces of 147 and $319 \mathrm{kN}$ for the frequencies of 19 and $28 \mathrm{~Hz}$, respectively. It is noteworthy that that a lower frequency will also reduce energy consumption (resulting in a fuel consumption reduction of up to $15 \%$ ) and machine wear.

The maximum contact forces between the soil and the drum, estimated from integrated roller measurements, are shown in Figure 7. A significant increase in contact force was apparent when utilising AFC, even though the centrifugal force of the drum was less than half of the force during conventional compaction. This decrease is due to the enhanced interaction between the drum and the soil at resonance, where the excitation takes advantage of the natural oscillation of the system. At a frequency far above resonance, the displacement and driving force oscillate out of phase and the force counteracts the displacement of the system.

Double-jumping can be analysed by observing the RMV, which depends to some extent on the characteristics of the accelerometer and the roller. For the equipment used in these tests, a value higher than approximately $6 \%$ could be classified as the roller being in a double-jump state. However, AFC gives rise to a slight frequency component at half the fundamental frequency due to resonance, which manifests as an increase of a few

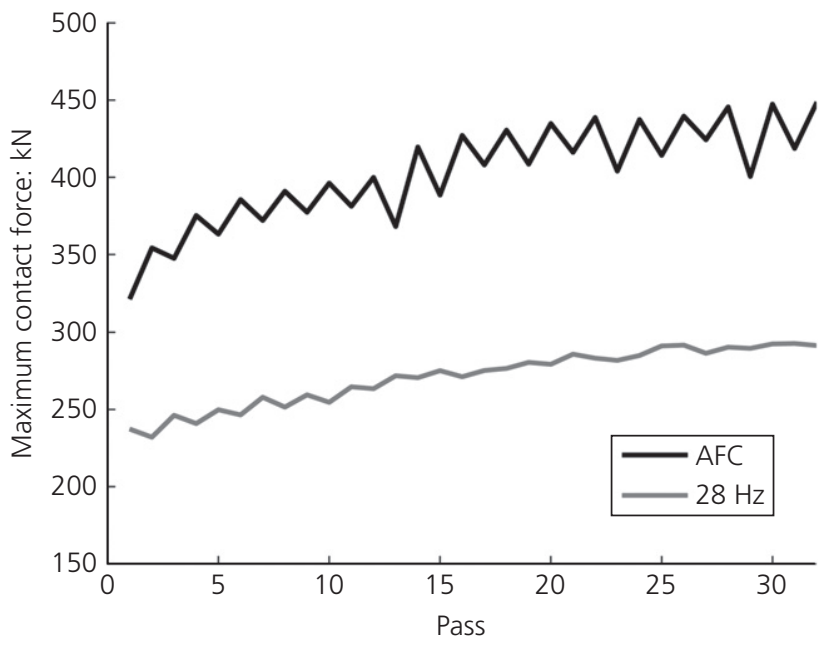

Figure 7. Average of maximum contact force for all passes 


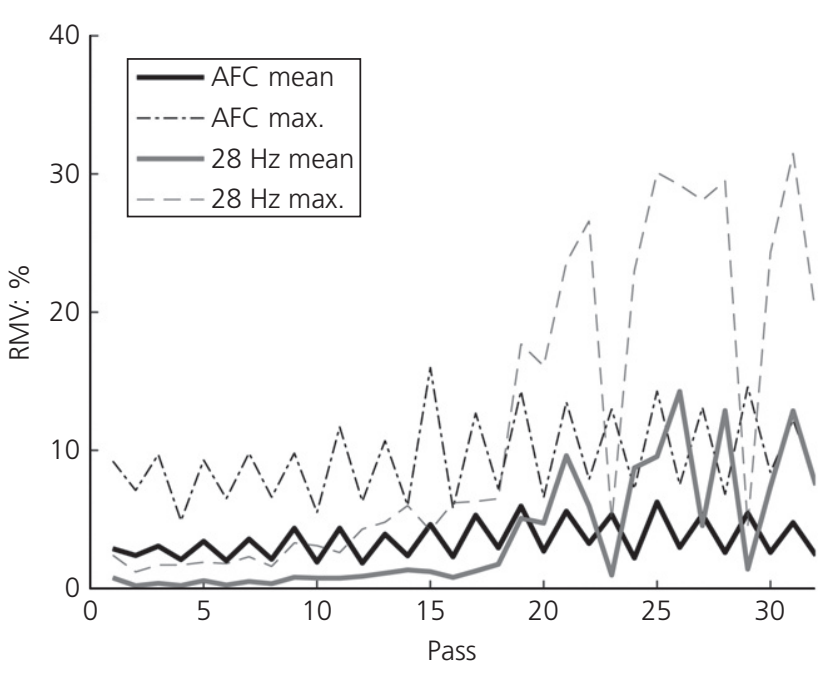

Figure 8. Average and maximum RMV for all passes

percent in the RMV throughout the entire compaction process (see Equation 1). This should not be mistaken for doublejumping. Figure 8 shows the RMV progress for all passes; a slightly enhanced value in the AFC data can be observed. No apparent increase in the RMV, in combination with no observed double-jump occurring during operation (double-jumping is directly apparent during operation as it drastically changes the dynamic behaviour of the roller), is a clear indication that the roller was in a state of either continuous contact or periodic loss of contact during all passes. For the fixed-frequency compaction, however, double-jumping started to occur at some locations during pass 19 and was apparent on most of the embankment during pass 21, which was confirmed by the dynamic behaviour during operation. In practice, the operator would abort compaction with a high amplitude after pass 21 at the latest and switch to a low amplitude (in the present tests, double-jumping was ignored in order to obtain comparable results between AFC and the fixed-frequency compaction at $28 \mathrm{~Hz}$ ). Alternatively, a few more passes could be conducted at a lower frequency if the roller allowed manual frequency adjustment. However, the lowest frequencies available on conventional rollers are still far above those utilised by the AFC system, and the lowest manually available frequency would soon cause double-jumping again due to the narrow frequency range available on most rollers. It should be noted that double-jumping is not correlated with the maximum compaction potential of the material, but rather with the operating conditions. The implication of the results shown in Figure 8 is that AFC facilitates more passes at high amplitude and thus a higher degree of compaction can be reached.

\subsection{Deformation measurements}

To some extent, settlement is related to compaction (Wersäll and Larsson, 2013). However, settlement also includes the components related to lateral soil displacement (especially

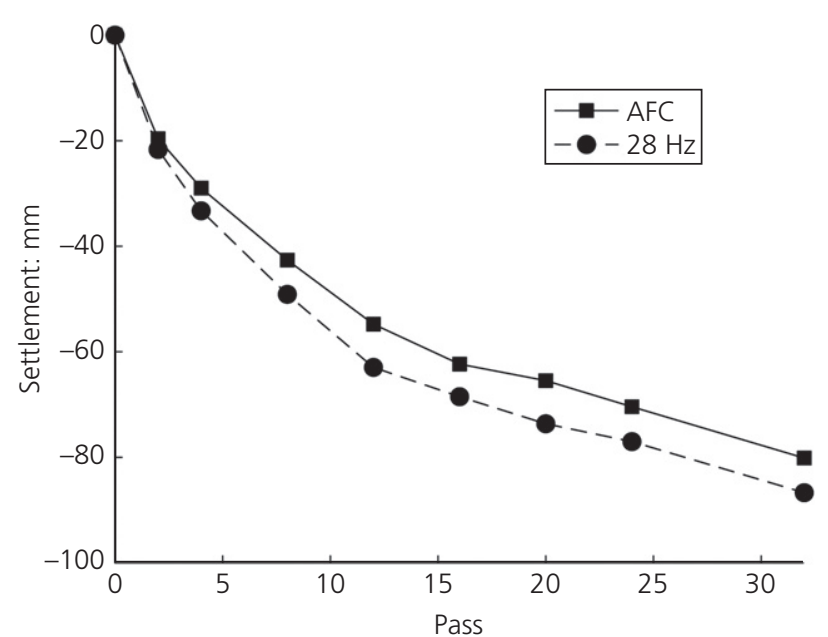

Figure 9. Average settlement of embankment surface

when the surrounding material is uncompacted) and to settlement at the subsoil level. The latter component can be assumed to be quite significant in this study due to the heavy rains that preceded the tests, which saturated the silty till below the embankment. The average settlements of the embankment surface are shown in Figure 9. The total settlement was larger for the fixed-frequency compaction than for AFC. However, this result is not sufficient to conclude a comparison of the relative degree of compaction between the two methods. Previous tests have shown that a high frequency transfers more compaction energy to the bottom of the layer (Wersäll et al., 2017). This is believed to relate to the greater number of impacts per unit length, and implies that settlement of the subsoil will be higher for a fixed (high) frequency. While the progress of CCC values halts at around 22 passes (Wersäll et al., 2019), the settlement increases during the whole compaction process, implying that other mechanisms - apart from compaction - significantly influence the settlements.

The results of the deformation measurements by the two columns of Emu sensors buried in each compaction lane were averaged to reduce uncertainties. These results included only the vertical deformation of the rock-fill and did not include the settlement of the subsoil; they thus provide a more reliable measure of the obtained degree of compaction than the total settlement measurement. Figure 10 shows the total compression for each lane and compaction method, from the top coil to the bottom coil), providing the average deformation of the layer. The final compression of the individual columns after 32 passes was 6.5 and $7 \cdot 1 \%$ for AFC and 5.6 and $6.3 \%$ for the fixed frequency compaction at $28 \mathrm{~Hz}$. Compaction at resonance by AFC resulted in significantly higher deformations than fixed-frequency compaction at $28 \mathrm{~Hz}$ when considering the full $1 \mathrm{~m}$ layer of rock-fill. A comparison of Figure 10 and Figure 9 yields the conclusions that compaction is better with AFC and that fixed-frequency compaction gives rise to larger settlements in the 


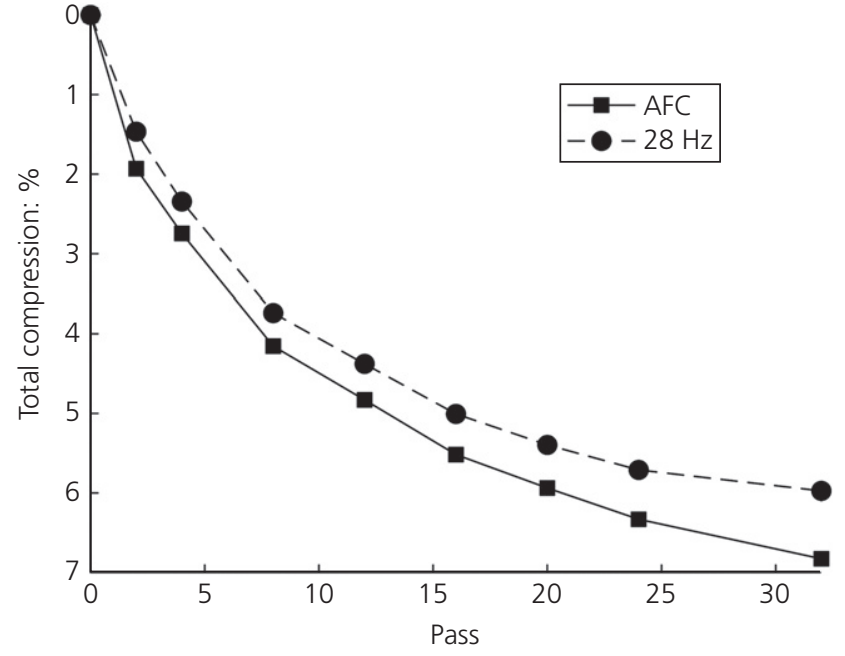

Figure 10. Total compression as measured by Emu sensors

subsoil. The latter may be beneficial in layer-wise embankment construction, as discussed below, but additional settlements in the subsoil may be unwanted.

The depth-dependent deformation was obtained by analysing the change in distance between individual coils. Figure 11(a) shows the compression profiles after two passes, where the depth of each data point corresponds to the midpoint of the pair of coils for which the compression was measured. The compression profiles after 32 passes are shown in Figure 11(b); they are similar in shape to the profiles obtained after two passes, but are more developed. At the bottom of the layer, a slight increase in compression was obtained for the fixedfrequency compaction, in line with previous tests (Wersäll et al., 2017). The coil pair at $700 \mathrm{~mm}$ showed less deformation than the other sensors. However, this is most likely the result of the placement procedure, in which the embankment was placed in two sub-layers and construction traffic caused initial compaction. These points should therefore be interpreted with caution. The major difference between AFC and the fixedfrequency compaction occurred at the top of the layer. Compaction by AFC provided a large deformation - and thus a large degree of compaction - at the top of the layer, while compaction at $28 \mathrm{~Hz}$ produced a poorly compacted material close to the embankment surface. A poor result at the surface during conventional compaction has been a well-known fact for many years (e.g. D'Appolonia et al., 1969) and is the reason why static passes are normally required after vibratory compaction has been finalised. The results from these tests suggest that supplementary static passes can be avoided when using AFC, thereby further reducing the total number of passes. Enhanced compaction at the surface for AFC is the result of optimised interaction between the drum and the soil at resonance. At higher frequencies, the counteraction of the drum and the soil results in compaction energy being consumed to loosen the

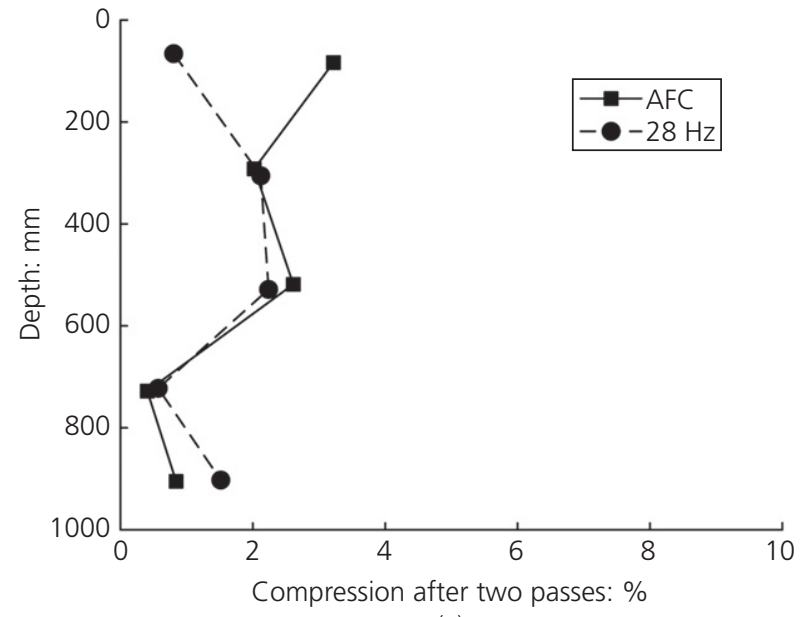

(a)

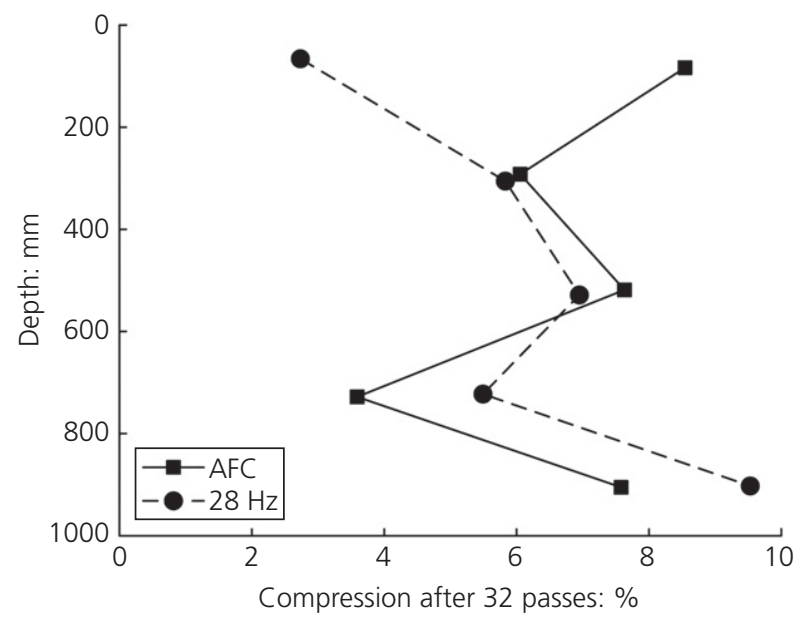

(b)

Figure 11. Compression profiles measured by Emu sensors (average of two columns): (a) after two passes; (b) after 32 passes

soil, which is the reason for the poor compaction at the surface. It is likely that the increased compaction effect that occurs at greater depths with the fixed-frequency compaction, which manifested as subsoil settlement in the current tests, would compact the loosened zone of the preceding layer to some extent in the case of layer-wise embankment construction. It is not possible to quantify the extent of this effect based on the results of this study. However, it is reasonable to assume that a somewhat loosened zone would remain, resulting in an inhomogeneous compaction profile with built-in stress and stiffness gradients that may cause rearrangement and settlement over time. Poor performance of compacted material due to vertical variation of properties has been reported recently by Titi et al. (2018). Therefore, the results presented in Figure 11 suggest that AFC will have a beneficial effect on the long-term behaviour of an embankment. Considering the enhanced compaction of AFC close to the surface and the slightly increased depth effect when using a high frequency, it is possible that combining the two methods could produce an optimised compaction result, 
Table 1. Stiffness measurements by PLTs and FWD measurements

\begin{tabular}{lccc} 
& \multicolumn{2}{c}{ Stiffness: $\mathbf{M P a}$} & \\
\cline { 2 - 3 } & AFC & $\mathbf{2 8 ~ H z}$ & $\begin{array}{c}\text { AFC stiffness/28 } \\
\text { stiffness: \% }\end{array}$ \\
\hline PLT (8 passes) & 108 & 105 & 103 \\
PLT (32 passes) & 157 & 135 & 116 \\
FWD 30 kN (32 passes) & 197 & 168 & 117 \\
FWD 50 kN (32 passes) & 201 & 170 & 118
\end{tabular}

although doing so would increase fuel consumption in comparison with solely using AFC. However, this is a topic for future research.

\subsection{Spot tests}

Stiffness measurements on the surface are shown in Table 1 . All the measurements were taken at different spots along the centre of each lane in order to obtain representative averages. After eight passes, the PLTs showed a very similar stiffness for both methods, implying that the variation in compaction at the surface does not greatly influence the stiffness of a moderately compacted embankment. After 32 passes, however, the part of the embankment compacted using AFC showed a $16 \%$ higher stiffness, on average, than the conventionally compacted part. The FWD measurements gave similar results, with $17 \%$ and $18 \%$ higher stiffness for loads of $30 \mathrm{kN}$ and $50 \mathrm{kN}$, respectively. All the stiffness measurements thus consistently indicate that the surface stiffness was greater after 32 passes when compaction was conducted with AFC, implying that there is a significant influence from the top of the embankment. Again, it should be noted that the greater stiffness was obtained with less than half of the centrifugal force and with a considerable reduction in fuel consumption.

\subsection{Fuel consumption and machine wear}

A reduced frequency implies a lower required power from the engine to run the vibratory part of the roller. Measurements at the Dynapac testing facility in Karlskrona, Sweden, showed that AFC reduced the power of the vibratory drum by close to $35 \%$, owing to the lower frequency. However, the power required for propulsion, steering, air conditioning compressor and so on was not affected. The resulting total power reduction was $10-15 \%$. Since fuel consumption is directly related to energy consumption, and thus power, this was reduced by the same amount, thus also reducing environmental impacts.

Machine wear is affected by the number of cycles. Since AFC reduces the frequency of operation, the number of cycles is reduced, which has a beneficial impact on bearings and hydraulics, especially on the risk for fatigue of various components. Furthermore, the wear of bearings is affected by the centrifugal force, which is reduced significantly when utilising the AFC system, as discussed earlier. It is thus reasonable to assume that the wear of the vibratory parts would be reduced to a large extent, implying an improved lifespan of the machine. However, this effect cannot be quantified until the system has been in use for a longer period of time.

\section{Conclusions}

A new method for vibratory roller compaction using AFC was evaluated in field experiments on a trial rock-fill embankment with a height of $1 \mathrm{~m}$ and compared with fixed-frequency conventional compaction. Various measurement techniques were used, combining roller-integrated systems, buried sensors and spot tests. The main results from these measurements are as follows.

- The continuously adjusted frequency by the AFC system, corresponding to the resonant frequency, was approximately $9 \mathrm{~Hz}$ lower than the fixed standard frequency.

- The centrifugal force was reduced by more than half when using AFC, due to the lower compaction frequency.

- The displacement amplitude and the contact force between the drum and the soil increased significantly with AFC, despite its lower energy consumption, indicating an enhanced interaction in the drum-soil interface.

- AFC significantly improved compaction close to the surface, as opposed to the loose zone obtained during conventional compaction, thus reducing or eliminating the need for subsequent static passes.

- The total compression of the $1 \mathrm{~m}$ layer was greater for AFC than for conventional compaction.

- Compaction at a fixed (high) frequency resulted in slightly enhanced compaction at greater depths, which also resulted in greater settlement of the subsoil.

- The use of AFC resulted in a more homogeneously compacted embankment, which may have a positive effect on its long-term behaviour.

- The surface stiffness from spot testing was similar for both methods after eight passes. After 32 passes, however, AFC produced a significantly higher stiffness.

- Double-jumping can be avoided by using AFC due to the reduced frequency and facilitates additional passes at high amplitude.

The enhanced interaction between the drum and the soil is connected to optimisation of the dynamic system to its most efficient state in order to produce large deformations at a low energy consumption - that is, resonance. At higher frequencies, the applied force counteracts the displacement while consuming an excessive amount of energy. The results from this study clearly indicate the beneficial effects on an embankment when compacting at the resonant frequency, and further indicate that automatic frequency adjustment is practically applicable without major technical difficulties. In addition to the benefits related to the embankment and the possible reduction in the total number of passes, AFC significantly reduces fuel consumption and thus environmental impact, while improving the lifespan of the roller. 


\section{Acknowledgements}

This project was funded by the Swedish Transport Administration, Better Interaction in Geotechnics (BIG), the Development Fund of the Swedish Construction Industry (SBUF), Dynapac Compaction Equipment AB and Peab AB.

\section{REFERENCES}

Adam D (1996) Flächendeckende Dynamische Verdichtungskontrolle (FDVK) mit Vibrationswalzen. $\mathrm{PhD}$ thesis, University of Vienna, Vienna, Austria (in German).

Anderegg R (2000) ACE Ammann Compaction Expert - automatic control of the compaction. In Compaction of Soils and Granular Materials (Gomes Correia A and Quibel A (eds)). Presses de l'Ecole Nationale des Ponts et des Chaussees, Paris, France, pp. 83-89.

Anderegg R and Kaufmann K (2004) Intelligent compaction with vibratory rollers: feedback control systems in automatic compaction and compaction control. Transportation Research Record 1868: 124-134.

Anderegg R, Von Felten DA and Kaufmann K (2006) Compaction monitoring using intelligent soil compactors. In GeoCongress 2006: Geotechnical Engineering in the Information Technology Age, Georgia, USA (DeGroot DJ, DeJong JT, Frost D and Baise LG (eds)). ASCE, Reston, VA, USA, pp. 1-6.

Arnold M and Herle I (2009) Comparison of vibrocompaction methods by numerical simulations. International Journal for Numerical and Analytical Methods in Geomechanics 33(16): 1823-2838.

Bernhard RK (1952) Static and dynamic soil compaction. Highway Research Board Proceedings 31: 563-592.

Cai J, Gao Q, Chun H, Cai H and Nantung T (2019) Spatial autocorrelation in soil compaction and its impact on earthwork acceptance testing. Transportation Research Record 2673: 332-342.

Converse FJ (1953) Compaction of Sand at Resonant Frequency. American Society for Testing and Materials, West Conshohocken, PA, USA, Special Technical Publication 156, pp. 124-137.

D'Appolonia DJ, Whitman RV and D'Appolonia E (1969) Sand compaction with vibratory rollers. Journal of the Soil Mechanics and Foundations Division 95(1): 263-284.

DIN (Deutsches Institut für Normung) (2012) DIN 18134:2012-04 Soil - Testing procedures and testing equipment - Plate load test. DIN, Berlin, Germany.

Dobry R and Whitman RV (1973) Compaction of Sand on a Vertically Vibrating Table. American Society for Testing and Materials, West Conshohocken, PA, USA, Special Technical Publication 523 , pp. $156-170$.

Forssblad L (1965) Investigations of Soil Compaction by Vibration. Acto Polytechnica Scandinavica, Civil Engineering and Construction Series No. 34. Royal Swedish Academy of Engineering Sciences, Stockholm, Sweden.

Imran AS, Barman M, Commuri S, Zaman M and Nazari M (2018) Artificial neural network-based intelligent compaction analyzer for real-time estimation of subgrade quality. International Journal of Geomechanics 18(6): 04018048.

Janoo V, Irwin L, Knuth K, Dawson A and Eaton R (1999) Use of inductive coils to measure dynamic and permanent pavement strains. Proceedings of the 1st International Conference on Accelerated Pavement Testing, Reno, NV, USA, pp. 18-20, CD-ROM.

Liu D, Li Z and Lian Z (2014) Compaction quality assessment of earth-rock dam materials using roller-integrated compaction monitoring technology. Automation in Construction 44: 234-246.

Liu D, Lin M and Li S (2016) Real-time quality monitoring and control of highway compaction. Automation in Construction 62: 114-123.

Massarsch KR (1991) Deep Soil Compaction Using Vibratory Probes. American Society for Testing and Materials, West Conshohocken, PA, USA, Special Technical Publication 1089, pp. 297-319.
Massarsch KR and Fellenius BH (2002) Vibratory compaction of coarsegrained soils. Canadian Geotechnical Journal 39(3): 695-709.

Meehan CL, Cacciola DV, Tehrani FS and Baker III WJ (2017) Assessing soil compaction using continuous compaction control and locationspecific in situ tests. Automation in Construction 73: 31-44.

Mooney MA and Rinehart RV (2007) Field monitoring of roller vibration during compaction of subgrade soil. Journal of Geotechnical and Geoenvironmental Engineering 133(3): 257-265.

Svensk Byggtjänst (2013) AMA Anläggning 13: Allmän Material - och Arbetsbeskrivning för Anläggningsarbeten. Svensk Byggtjänst, Västerås, Sweden (in Swedish).

Titi HH, Tabatabai H, Faheem A, Tutumluer E and Peters JP (2018) Spatial variability of compacted aggregate bases. Transportation Geotechnics 17: 56-65.

Wersäll C and Larsson S (2013) Small-scale testing of frequencydependent compaction of sand using a vertically vibrating plate. Geotechnical Testing Journal 36(3): 1-10.

Wersäll C, Larsson S, Rydén N and Nordfelt I (2015) Frequency variable surface compaction of sand using rotating mass oscillators. Geotechnical Testing Journal 38(2): 1-10.

Wersäll C, Nordfelt I and Larsson S (2017) Soil compaction by vibratory roller with variable frequency. Géotechnique 67(3): 272-278, https://doi.org/10.1680/jgeot.16.P.051.

Wersäll C, Nordfelt I and Larsson S (2018a) Resonant roller compaction of gravel in full-scale tests. Transportation Geotechnics 14: 93-97.

Wersäll C, Nordfelt I and Larsson S (2018b) Roller compaction of rock-fill with automatic frequency control. Proceedings of Anniversary Symposium - 40 Years of Roller Integrated Continuous Compaction Control (CCC), Vienna, Austria (Adam D and Larsson S (eds)). Technische Universität Wien, Institut für Geotechnik, Vienna, Austria, pp. 97-100.

Wersäll C, Åkesson F and Persson A (2019) Dynamic roller characteristics and $\mathrm{CCC}$ using automatic frequency control. Proceedings of International Intelligent Construction Technologies Group Conference 2019, Beijing, China.

White DJ, Jaselskis EJ, Schaefer VR et al. (2004) Field Evaluation of Compaction Monitoring Technology: Phase I. Final Report. Center for Transportation Research and Education, Iowa State University, Ames, IA, USA, Iowa DOT Project TR-495.

Youd TL (1972) Compaction of sands by repeated shear straining. Journal of the Soil Mechanics and Foundations Division 98(7): 709-725.

Zhang Q, Liu T, Zhang Z et al. (2019a) Compaction quality assessment of rockfill materials using roller-integrated acoustic wave detection technique. Automation in Construction 97: 110-121.

Zhang Q, Liu T, Zhang Z et al. (2019b) Unmanned rolling compaction system for rockfill materials. Automation in Construction 100: 103-117

\section{How can you contribute?}

To discuss this paper, please email up to 500 words to the editor at journals@ice.org.uk. Your contribution will be forwarded to the author(s) for a reply and, if considered appropriate by the editorial board, it will be published as discussion in a future issue of the journal.

Proceedings journals rely entirely on contributions from the civil engineering profession (and allied disciplines). Information about how to submit your paper online is available at www.icevirtuallibrary.com/page/authors, where you will also find detailed author guidelines. 